\title{
O perfil do desenvolvimento motor de crianças praticantes e não praticantes do futsal
}

\author{
Motor development profile of a child who practicing futsal and non-practitioners child
}

\author{
Gustavo Armando Aparecido da Silva Pereira ${ }^{1}$ \\ Vitor Luiz de Andrade ${ }^{1, *}$
}

\section{Resumo}

Objetivo: O estudo objetivou avaliar o desempenho psicomotor de crianças praticantes e não praticantes do futsal e verificar o eventual benefício do esporte. Métodos: Participaram do estudo 20 crianças do sexo masculino de 7 anos distribuídas em dois grupos. O grupo que não pratica futsal (GNP) foi selecionado em uma escola pública de ensino fundamental I, as crianças praticantes de futsal foram recrutadas em projeto social (GP). Os dados foram coletados através da bateria de testes psicomotores proposta por Rosa Neto. Resultados: O GP $(100,8 \pm 6,2$ meses) é significativamente melhor na idade motora geral em relação ao GNP (92,6 $\pm 3,2$ meses; $t=1,734 ; p=0,0003)$. Os resultados sugerem que o esporte contribui positivamente para o desenvolvimento psicomotor das crianças. Conclusões. Mesmo sendo praticado de forma sistematizada e com movimentos específicos do esporte, o futsal oferece diversidade de movimentos, com alto índice de estímulo neuromuscular, o que pode justificar a diferença entre os grupos.

Palavras-chave: desenvolvimento motor, psicomotricidade, futsal.

\begin{abstract}
Objective: The aim of this study was to evaluate the psychomotor performance of children practicing and not practicing futsal and to prove possible benefit of sport. Methods: 20 male 7-year-old boys were separated in two groups. The group that does not practice futsal (NPG) was selected in a public elementary school. The futsal practicing children were recruited in social project (FP). The data were collected through the battery of psychomotor tests proposed by Rosa Neto. Results: FP is significantly better in general motor age in relation to NPG $(t=1.734 ; p=0.0003)$. The results suggest that sport contributes positively to children's psychomotor development. Conclusion: Although practiced in a systematic way and with specific movements of the sport, the futsal offers diversity of movements, with a high index of neuromuscular stimulus, which may justify the difference between the groups.
\end{abstract}

Key-words: motor development, psychomotricity, futsal.
Afiliação dos autores

${ }^{1}$ Centro Universitário UNIFAFIBEUNIFAFIBE, Bebedouro, São Paulo, Brasil.

${ }^{\star}$ Autor correspondente

Curso de Educação Fìsica do Centro Universitário UNIFAFIBE, Bebedouro, SP, Brasil.

e-mail:

vitor.luiz.de.andrade@gmail.com

Conflito de interesses

Os autores declararam não haver conflito de interesses.

Processo de arbitragem

Recebido: 03/06/2017 Aprovado: 15/03/2018 


\section{Introdução}

De acordo com a Sociedade Brasileira de Psicomotricidade, a psicomotricidade é a ciência que tem como objetivo de estudo o homem através do seu corpo em movimento e em relação ao mundo de forma interna e externa ${ }^{1}$. Está relacionada ao processo de maturação, onde o corpo é a origem das aquisições cognitivas, afetivas e orgânicas. É sustentada por três conhecimentos básicos: o movimento, o intelecto e o afeto ${ }^{1}$.

O desenvolvimento da psicomotricidade permite transformar o cérebro num órgão capaz de captar, integrar, armazenar, elaborar e expressar informação ${ }^{2}$. É uma ciência de extrema importância no âmbito dos estudos de desenvolvimento humano, desde o contexto psicológico até ao motor, ou seja, partindo da forma em que pensamos, movimentamos e agimos ${ }^{2}$. A descrição do processo de desenvolvimento motor da criança conta com três aspectos: I: a sequência que não indica apenas aquilo que a criança pode aprender, mas às suas necessidades; II: a velocidade, que varia de criança para criança; III: a interdependência entre as mudanças de habilidades simples para as complexas ${ }^{3,4}$.

Neste contexto, o esporte poderia ser um agente transformador permitindo que os três aspectos do desenvolvimento motor da criança sejam atingidos da melhor forma possível, no tempo adequado e necessário respeitando a individualidade biológica de cada criança ${ }^{1,4,5}$. Estudos demostram que o esporte promove além de benefícios fisiológicos e psicológicos pode acelerar o procedimento de maturação biológica ${ }^{6,7}$. A atividade sistemática proporciona aos seus praticantes um conhecimento e domínio sobre o corpo, o que permite que suas vivências sociais modernas sejam mais facilmente desenvolvidas e vividas.

Entretanto, atividades vigorosas e cíclicas não atraem crianças para a prática esportiva, por isso, o futsal como ferramenta de estudo pode, de acordo com os princípios da psicomotricidade, trazer contribuições significativas para 0 desenvolvimento psicomotor de crianças que praticam essa modalidade em relação às crianças que não realizam a prática esportiva. Por isso, o objetivo da presente investigação é verificar se existem diferenças no perfil do desenvolvimento motor de crianças que praticam futebol em relação às que não praticam.

\section{Métodos}

\section{Participantes}

Participaram dessa pesquisa 20 crianças do sexo masculino de 7 anos de idade, divididas em dois grupos. 10 crianças compuseram $\circ$ grupo não praticante de futsal (GNP) selecionadas em uma escola pública de ensino fundamental I e 10 crianças compuseram o grupo de praticantes de futsal (GP), todas recrutadas de um projeto social (Educando para o Esporte), ambos da cidade de Terra Roxa/SP. O tamanho amostral mínimo foi calculado por meio do software G*Power 3.1 (Düsseldorf, Germany), utilizando a referência de os valores observados do estudo ${ }^{9}$ que mais se aproximou aos interesses do presente estudo. O poder estatístico foi assumido como $95 \%$ $(p$-valor 0,05$)$ e o tamanho do efeito utilizado foi de 1,3211 . Neste modelo estatístico o número de participantes mínimo foi sete $(t$-valor crítico $=1,7823)$.

Como critérios de inclusão, os participantes do futsal deveriam estar frequentando regularmente os treinos no mínimo há 12 meses. Ao grupo de não praticantes do futsal, solicitou-se apenas crianças que nunca participaram de turmas de treinamento esportivo. Não foram incluídas crianças que praticavam mais de uma modalidade esportiva. Somente foi incluído no estudo aquele que apresentou o Termo de Consentimento Livre e Esclarecido assinado pelo pai, mãe ou responsável. Todos os procedimentos adotados foram aprovados pelo Comitê de Ética em Pesquisa em Humanos da Instituição.

\section{Delineamento da pesquisa e aplicação da ferramenta}

O instrumentos de coleta de dados foi a escala de desenvolvimento motor proposta por Rosa Neto ${ }^{10}$ que inclui protocolos para avaliar lateralidade dos pés, das mãos e dos olhos, seguindo das habilidades psicomotoras, motricidade fina, motricidade global, equilíbrio, esquema corporal, organização espacial e organização temporal.

As idades dos participantes foram utilizadas em meses e pareadas o mais próximo possível em ambos os grupos, buscando evitar que a diferença de vida influenciasse nos resultados, podendo assim, afetar negativamente na pesquisa.

Os testes iniciam com tarefas referentes à idade da criança, quando a mesma não conseguir realizar a tarefa, ocorre à regressão do teste correspondente à faixa etária até que ela efetue com sucesso. Ao avançar a tarefa correspondente a sua idade, passa-se para a próxima etapa até que a criança não consiga avançar mais. A marcação deve ser feita correspondente à tarefa que ela conseguiu fazer por último. Exemplo: A criança com 7 anos, começou com a tarefa para sete anos e foi até a atividade para 9 anos. Quando passou para 10 anos ela não conseguiu realizar, desta forma a pontuação (dada conforme o protocolo) será referente a 9 anos. A bateria de teste foi efetuada seguindo a sequência de provas motoras: motricidade fina, motricidade global, equilíbrio, esquema corporal, organização espacial e organização temporal.

Protocolos de resultados foram tabulados por Idade Cronológica (IC) em meses e pelas respectivas idades motoras (IM), em cada tarefa, cujo resultado é obtido com base nas tabelas normativas. A idade negativa e idade positiva são obtidas pela subtração da idade motora geral pela idade cronológica. Os valores serão positivos quando a idade motora geral apresentar valores numéricos superiores à idade cronológica, expressa em meses.

Os cálculos da idade motora geral (IMG - Equação 1) e quociente motor geral (QMG - Equação 2) são expressos pelas seguintes fórmulas:

$$
\text { Equação 1: }(I M G)=I M 1+I M 2+I M 3+I M 4+I M 5+I M 6 / 6 .
$$

$$
\text { Equação 2: }(\mathrm{QMG})=(\mathrm{IMG} / \mathrm{IC}) \text { * } 100 .
$$

\section{Análise estatística}

A normalidade dos dados foi confirmada por meio do teste de Shapiro-Wilk. Após a confirmação da normalidade, os dados foram apresentados em média \pm desvio padrão. $O$ teste $t$ de Student foi realizado para diferenciar as médias de idade motora geral e quociente motor geral. Para todos os casos o nível de significância foi de $p<0,05$.

\section{Resultados}

A IMG foi diferente entre os grupos, o GP apresentou 100,8 $\pm 6,2$ meses e o GNP de $92,6 \pm 3,2$ meses $(p<0.001)$. A idade cronológica não foi diferente entre os grupos (93,6 $\pm 5,4$ meses para GP e 93,7 $\pm 2,7$ meses para GNP). O GP obteve um score de idade positiva (7,2 $\pm 0,5$ meses) e quanto o GNP obteve um score negativo de idade ( $-1 \pm 0,2$ meses). O QMG foi de 107,7 \pm 4,5 meses no GP e $99 \pm 0,2$ no GNP $(p<0.01)$.

\section{Discussão}

Os principais achados do trabalho revelam a relevante importância da prática esportiva no desenvolvimento motor da criança. De acordo com os resultados, o perfil do desenvolvimento motor de crianças praticantes de futsal foi melhor que crianças que não praticavam, demonstrando a efetividade do esporte em desenvolver o fator físico relacionado a psicomotricidade.

Para Gallahue e Ozmun ${ }^{11}$, o processo de desenvolvimento motor acontece em quatro fases. A primeira fase é a motora reflexiva caracterizada por reflexos que são as primeiras formas de movimento humano (quatro meses a um ano de idade). A segunda fase é chamada de motora rudimentar (um e a dois anos de idade) na qual ocorrem os primeiros movimentos rudimentares (pouca coordenação). A terceira fase é apontada de movimentos fundamentais (entre dois a sete anos de idade). A última fase do desenvolvimento motor é da especialização motora que acontece a partir dos sete anos de idade. Neste contexto, quando estudadas crianças de sete anos de idade, praticantes e não praticantes de futsal. Encontramos evidente mudança no perfil de desenvolvimento motor, caracterizada principalmente pela prática esportiva. 
O esporte pode promover muitos benefícios para a formação integral da criança, tais como: desenvolvimento das capacidades de desempenho corporal e motor, bem como aspectos relacionados à cooperação, convivência, participação, inclusão e satisfação ${ }^{12}$. Tomando a base do conhecimento gerado na Educação Física, o esporte revela muita importância no desenvolvimento motor da criança. Embora outros estudos devam ser realizados em outros esportes para caracterizar os efeitos do esforço no desenvolvimento motor. Os resultados da presente investigação mostram que os praticantes de futsal têm probabilidade de obter maiores níveis de coordenação motora.

Os resultados obtidos no estudo descritivo sobre o perfil do desenvolvimento motor de crianças praticantes e não praticantes do futsal mostraram que a atividade esportiva contribui positivamente no desenvolvimento psicomotor das crianças. Mesmo sendo praticado de forma sistematizada e com movimentos específicos do esporte. Portanto, os programas de iniciação esportiva poderão ser importantes para a formação da criança, desenvolvimento de capacidades motoras e desempenho corporal, além de ajudar na integração da criança com a sociedade. Entretanto, estes resultados devem ser tomados com cautela, haja vista que a especialização precoce parece contribuir negativamente como um grande risco do esporte competitivo durante a iniciação esportiva. A busca incessante pelo prestígio conduz professores e familiares a exporem as crianças a situações de grande exigência e tensão, de treinamentos intensivos e precoces em busca de altos rendimentos pode ser muito danoso e prejudicial para a vida do cidadão em sociedade ${ }^{13}$.

\section{Conclusão}

O presente estudo teve como proposito uma análise descritiva e comparativa em relação ao desempenho motor de crianças com idade de 7 anos, praticantes e não praticantes do futsal e, comprovar o eventual benefício do esporte. Pode-se concluir que o desenvolvimento motor de crianças praticantes de futsal é melhor, por isso, destaca-se a prática esportiva como agente fundamental para desenvolvimento motor de crianças.

\section{Referências}

1. Lussac RMP. Psicomotricidade: história, desenvolvimento, conceitos, definições e intervenção profissional. efdeportes Revista Digital - Buenos Aires. 2008; 13(126).

2. Oliveira GCC. Psicomotricidade: educação e reeducação em um enfoque psicopedagógico. Petrópolis: Vozes; 1997.

3. Santos S, Dantas L, Oliveira JA. Desenvolvimento motor de crianças, de idosos e de pessoas com transtornos na coordenação. Rev paul Educ Fís. 2004; 18.

4. Ferreira V. Educação física, interdisciplinaridade, aprendizagem e inclusão. Rio de Janeiro: Sprint; 2006.

5. Rodrigues D. A Educação Física perante a Educação Inclusiva: reflexões conceptuais e metodológicas. Boletim da Sociedade Portuguesa de Educação Física. Lisboa; 2001. p. 73-81.

6. Bernink MJ, Erich WB, Peltenburg AL, Zonderland ML, Huisveld IA. Height, body composition, biological maturation and training in relation to socioeconomic status in girl gymnasts, swimmers, and controls. Growth. 1983; 47(1): 1-12.

7. Falgairette G, Bedu M, Fellmann N, Van-Praagh E, Coudert J. Bioenergetic profile in 144 boys aged from 6 to 15 years with special reference to sexual maturation. Europeanjournalofappliedphysiologyandoccupationalphysiology. 1991; 62(3): 151-6.

8. Lazzoli JK, Nóbrega ACLd, Carvalho Td, Oliveira MABd, Teixeira JAC, Leitão $\mathrm{MB}$, et al. Atividade física e saúde na infância e adolescência. Revista Brasileira de Medicina do Esporte. 1998; 4: 107-9.

9. Leandro ALGC. Contributo da reeducação psicomotora para ultrapassar as dificuldades de aprendizagem de um aluno com dislexia. (Estudo de Caso). Lisboa: Escola Superior de Educação João de Deus; 2013.

10. Rosa Neto F. Manual de Avaliação Motora. Porto Alegre: ARTMED; 2002.

11. Gallahue DL, Ozmun JC, Goodway JD. Compreendendo O Desenvolvimento Motor - 7ed: Bebês, Crianças, Adolescentes e Adultos: AMGH Editora; 2013.

12. Filgueira FM. Futebol: uma visão da iniciação esportiva: Ribergráfica; 2004.

13. Fechio JJ, Cichowicz FDA, Castro NM, Alves H. Especialização esportiva precoce: uma revisão. EFdeportes, Revista Digital Buenos Aires 2012 17(169). 